\title{
Elektrische Maschinen und Antriebe
}

\author{
H. Neudorfer OVE, VDE, IEEE
}

Online publiziert am 13. Februar 2015

(c) Springer Verlag Wien 2015

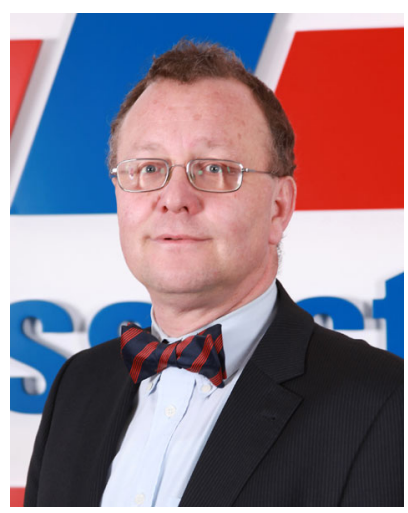

Univ.-Prof. Dr. phil. Dr. techn. habil. Harald Neudorfer
Auf dem Fachgebiet der elektrischen Maschinen und Antriebe gibt es nach wie vor weitere neue Entwicklungen, die vor allem auf die Effizienz der Systeme eingehen. Obwohl die Grundprinzipien für elektrische Maschinen seit ca. 150 Jahren bekannt sind, können aufgrund von neuen Materialien, innovativen Regelungsalgorithmen und speziellen Anpassungen für den Anwendungsfall immer wieder Fortschritte erzielt werden. Große Anstrengungen in der Weiterentwicklung von elektrischen Antriebssystemen richten sich auch auf das Ziel, die Wirkungsgrade zu verbessern. Dabei werden auch bei Maschinen kleinerer Leistungsklassen die Einzelverluste noch präziser berechnet. Dies ist allerdings nur dann möglich, wenn der elektrische Antrieb als multi-physikalisches Gesamtsystem betrachtet wird, bei dem neben der elektromagnetischen Auslegung auch die mechanische, thermische, strömungstechnische und geräuschtechnische berücksichtigt wird. Daraus ergibt sich, dass nicht nur klassische Elektrotechniker, sondern auch Maschinenbauer, Strömungstechniker oder ganz allgemein Physiker in das Design des elektrischen Antriebes eingebunden werden. Ein weiterer wesentlicher Punkt für Neuentwicklungen bei elektrischen Maschinen und Antrieben ist der zwingende Wunsch nach Kostenreduzierung. Um diesem wirtschaftlichen Druck bezüglich Kostenoptimierung zu entsprechen, werden bei einigen nachvollziehbaren Anwendungen sogar Performanceeinbußen im technischen Bereich in Kauf genommen. Bei Maschinentypen mit großen Stückzahlen, wie zum Beispiel in der Automobilindustrie, kommt bei der Auslegung neben den oben angeführten Experten auch den Fertigungstechnikern große Bedeutung zu. Ihre Aussage über eine kostenoptimale Fertigungstechnologie kann das Maschinendesign wesentlich beeinflussen.

Die Weiterentwicklung elektrischer Maschinen und deren optimierte Auslegung wird auch von immer aufwändigeren Finite Elemente-Programmen beeinflusst. Es kann durchaus behauptet werden, dass die Auslegung einer elektrischen Maschine im Forschungs- und Entwicklungsbereich, aber auch bei der industriellen Anwendung, ohne Finite Elemente-Berechnung nicht mehr ausgeführt wird.

Durchaus erfreulich ist die Tatsache, dass die Anzahl der Studierenden, die sich für das Gebiet der elektrischen Maschinen und Antriebe interessieren, in den letzten Jahren merkbar gestiegen ist. Die vor 10 bis 15 Jahren gängige Meinung, dass auf dem Gebiet der elektrischen Maschinen keine großartigen Weiterentwicklungen mehr zu erwarten sind, hat sich nicht bewahrheitet. Trotz längst bekannter Grundprinzipien elektrischer Maschinen stiegen laufend die Varianten von Anwendungen für elektrische Systeme. Zwei typische aktuelle Beispiele sind die Themenkreise "Elektromobilität" und "Hybridisierung". Diese durchaus auch sozialpolitischen Tendenzen werden von den Studierenden wahrgenommen und somit das Teilgebiet der Elektrotechnik "Elektrische Maschinen und Antriebe" vermehrt als Studienzweig angenommen.

Durch die sehr große Resonanz seitens der angeschriebenen Autoren wurden für das vorliegende Themengebiet "Elektrische Maschinen und Antriebe" eine Vielzahl an Beiträgen eingesendet. Nach Rücksprache mit der e\&i-Schriftleitung haben wir uns entschlossen, im Heft 1/2015 insgesamt 13 begutachtete Originalarbeiten aufzunehmen. Für die große Anzahl an hochwertigen Papers und die Bereitschaft des Herausgebers, ein Heft mit so vielen Beiträgen zu gestalten, möchte ich mich als Heftkoordinator bei allen Beteiligten sehr herzlich bedanken. Es ist dies als ein starkes Lebenszeichen aller mit dem Themengebiet befassten Institute an den Technischen Universitäten und in der einschlägigen Industrie zu werten.

Die aktuelle Ausgabe der vorliegenden Zeitschrift beinhaltet folgende Beiträge:

Weiss, Polt, Geschrey und Schrödl beschreiben stromrichterbedingte Verluste am Beispiel eines permanenterregten Synchrongenerators bei unterschiedlichen Schaltfrequenzen und den Unterschied bei oberflächenmontierten und vergrabenen Magneten.

Schmidt untersucht mittels numerischer Analyse permanenterregte Synchronmaschinen (PSM) mit Bruchloch-Zahnspulenwicklungen und stellt die Einflüsse der Permanentmagnete und der Statorströme in Abhängigkeit von Rotorwinkellage und unterschiedlicher Sättigungszustände näher dar.

Neubauer und Neudorfer thematisieren in ihrem Beitrag ebenfalls das Thema PSM mit Zahnspulenwicklungen. Vorgestellt wird ein neuartiger Traktionsgenerator mit geschrägten Nuten, mit dem kurze axiale Länge bei hoher Leistungsdichte realisiert wird.

Lehr, Reis und Binder vergleichen Axial- und Radialflussmaschinen für den Einsatz in Radnabenantrieben und gehen auch mit Hilfe der Finite Elemente-Methode unter besonderer Berücksichtigung der Lochzahl auf die Auslegung der Maschinen ein.

Misir, Dobbert und Ponick stellen eine analytisch numerische Berechnung des magnetischen Luftspaltleitwertes für Rotoren einer Schenkelpolsynchronmaschine vor und vergleichen deren Abhängigkeit von unterschiedlichen Parametern.

Bacher und Mütze vergleichen Asynchronmaschinen (ASM) mit verteilten und konzentrierten Wicklungen. Der Einsatz von konzentrierten Wicklungen bringt Kostenersparnisse bei der Fertigung, aber auch einen erhöhten Anteil an höheren Harmonischen bei diesem Maschinentyp.

Herold, Franck, Böhmer, Schröder und Hameyer zeigen in ihrer Abhandlung ein transientes Simulationsmodel für lokale Kraftanregungen in elektrischen Antrieben. Damit ist es möglich, drehzahlund drehmomentdynamische Vorgänge zu bewerten.

Neudorfer, Harald, Traktionssysteme Austria GmbH, Brown Boveri Straße 1, 2351 Wiener Neudorf, Österreich (E-Mail: Harald.Neudorfer@traktionssysteme.at); Neudorfer, Harald, Technische Universität Darmstadt, Darmstadt, Deutschland; Neudorfer, Harald, Technische Universität Wien, Wien, Österreich 
Weber und Neudorfer verdeutlichen den multiphysikalischen Ansatz bei hochausgenützten Traktionsmaschinen und berechnen mit Hilfe der Computational Fluid Dynamics-Methode (CFD) die auftretenden Druckverluste und Strömungsverhältnisse sowie die vorherrschenden Temperaturen aufgrund des Ventilationssystems in elektrischen Maschinen.

Hettegger, Mann, Bíró und Ofner untersuchen die Strömungsbedingungen des Kühlmediums in Oberflächennähe und den Einfluss auf den Temperaturtransfer bei forcierter Konvektion. Die Berechnungsergebnisse mittels CFD werden mittels Particle Image Velocimetry (PIV) verifiziert.

Dinca und Schäfer gehen in ihrem Beitrag auf Kreisströme bei permanenterregten brushless DC Motoren (BLDC) mit Dreieckschaltung ein. Diverse harmonische Ströme werden meist aufgrund von speziellen Nut-/Polkombinationen, durch Asymmetrien von Rotor und Stator, Phasenasymmetrien sowie magnetische Sättigungen im Joch und in den Zähnen hervorgerufen.

Kreim und Schäfer bestimmen Umrichterverluste in permanenterregten Hilfsantrieben für Kraftfahrzeuge. Da diese Verluste für die thermische Auslegung benötigt werden, müssen diese neben allgemeiner Wechselmagnetisierung auch für allgemeine Drehmagnetisierung bestimmt werden.

Demmelmayr, Feischl, Troyer und Schrödl lassen in ihrem Beitrag die enge Kopplung der elektrischen Maschine mit dem speisenden Wechselrichter deutlich erkennen. Dabei werden hochdynamische Phasenstrommessungen für Traktionsantriebe mit anisotropen magnetoresistiven Sensoren (AMR) beschrieben. Die Ergebnisse werden mit Referenzmessungen kompensierender LEM-Stromwandler verglichen

Müllner, Neudorfer und Recheis beschreiben die Entwicklung eines mobilen Flywheels als Energiespeicher. Der nutzbare Energieinhalt von 900 Wh mit einer Spitzenleistung von 145 kW wird durch ein integriertes Rotorkonzept basierend auf einer synchronen Reluktanzmaschine für eine Drehzahl bis $40.000 \mathrm{~min}^{-1}$ realisiert.

Zum Abschluss des Vorwortes möchte ich mich noch bei allen Kolleginnen und Kollegen herzlich bedanken, die am Themengebiet "Elektrische Maschinen und Antriebe" forschen, sich dessen Weiterentwicklung widmen und ihr Wissen weitergeben. 